\title{
Surfacing the Organisation-in-the-mind
}

\author{
(C) 2012 Sandra Wilson
}

\begin{abstract}
The theory of the organisation-in-the-mind suggests that organisations live within us, are a part of our identity and are bound up with our inner emotional life. Connecting this theory to Berne's thinking on the structure and dynamics of organisations and groups it is possible through interpretative phenomenological analysis to conduct research into how what we hold in the unconscious impacts on professional relationships and performance. The outcome from research in this area is intended to highlight potential areas for development of competence in professional coaches.
\end{abstract}

\section{Background and Context}

\section{Coaching}

Coaching has become a mainstream offering in management and executive development functions. Engaging professional coaches to work with managers on their development has become a regular occurrence in large organisations across the UK and Europe (Peltier 2009). The role of coaching in organisations has evolved from its roots as the organisation's agent on performance mediation to the present focus on releasing potential through professional/personal development plans.

To keep pace with the increased sophistication professional development organisations have hired individuals to work with executives on their development plans. Until relatively recently coaches were always external to the organisation (Tyler 2000). This made sense given the original requirements of the role; coaching skills focussed on behavioural change, knowledge of organisational functioning, confidentiality, and, to varying degrees, an overarching professional identity that elevated the coach's credibility for both the individual and the organisation.

Into the maturing arena of coaching comes the predictable, but not entirely obvious, emergence of the internal coach. The role of the internal coach has achieved recognition in many large organisations as valuable in its own right. It does not, generally, represent a professional's full-time responsibilities, but, where it exists, the role itself has achieved enough legitimacy to elicit a development commitment essentially the same as would be expected with an external coach.

The simplest definition of internal coaching may be a tautology; being formally viewed by the organisational co-workers as a coach makes it so. The clarity and strength of the label, formalised under a job title, may be all that is needed to operate as an internal coach. However, wanting to be more descriptive and in keeping with an attempt to delineate the emerging role, the following definition which is used in the NHS in Scotland is offered: "Internal coaching is a one-to-one development intervention supported by the organisation and provided by a colleague of those being coached who is trusted to shape and deliver a programme yielding individual professional growth."

The important and obvious difference between the external and internal coach is that the internal coach is a fellow employee in the same organisation as those he or she coaches. The implications of this with particular regard to ethics were explored in Wilson's (2008) seminal paper "True Dilemmas" and St John Brooks (2009) research into ethical considerations for internal coaching.

The use of external coaches overcomes any hierarchical issues which may prevail when a senior manager or executive is offered coaching by someone further down the chain of command. It could be argued that external coaching relationships are "cleaner"; internal coaches may have multiple roles, relationships and interactions, both formal and informal, with those they coach. The situation may be confusing or raise concerns about trust and confidentiality if not handled with sensitivity and forethought.

Given their mutual trade-offs, even where an internal coaching function exists, however, there will always be managers and executives for whom external coaching is the better choice. This may be to do with the complexity of the personality involved, coach credibility, concerns about confidentiality or other factors.

\section{Theoretical Underpinning}

The organisation-in-the-mind 
The term "organisation in the mind" was part of the lingua franca of the Grubb Institute. In working with this term psychoanalysts were seeking to draw attention to the ways in which one might understand the behaviour of group members as reflecting and being governed by unconscious assumptions, images and fantasies held about the organisation. Shapiro and Carr (1991) went on to say:

"Any organisation is composed of diverse fantasies and projections of its members. Everyone who is aware of an organisation, whether a member or not, has a mental image of how it works. Though these diverse ideas are not often consciously negotiated or agreed upon among participants, they exist. In this sense, all institutions exist in the mind, and it is in interaction with these inthe-mind entities that we live".

Hutton, Balgazette and Reid (1997) in a subsequent paper formulated the idea as follows:-

"Organisation-in-the-mind is what the individual perceives in his or her head of how activities and relations are organised, structured and connected internally. It is a model internal to oneself, part of one's inner world, relying on the inner experiences of my interactions, relations and activities I engage in which give rise to images, emotions, values and responses in me, which may be consequently influencing my own management and leadership, positively or adversely. "Organisation-in-the-mind helps me to look beyond the normative assessments of organisational issues and activity, to become alert to my inner experiences and give richer meaning to what is happening to me and around $\mathrm{me}^{\prime \prime}$

With this concept in mind it is possible to start considering the issue or the extent to which the "organisation-in-the-mind" influences the coaching contract for both external and internal coaches and whether the influence is greater for one or the other.

\section{The Concept of the Psychological Contract}

The research topic aims to bring together the psychoanalytic concept of the "organisation-in-themind" with the Transactional Analysis concept of the psychological contract.

The research aims to ascertain the extent and nature of the differences in the psychological contract held by internal and external coaches with a focus on the "organisation-in-the mind". The research will seek to establish whether one or other type of coach can effect a "cleaner" contract with those they are coaching because they are less influenced by the culture they experience which influences their beliefs, attitudes and behaviours.

The concept of "cleaner" contracting is derived from my understanding that individuals pursue unconscious tasks alongside their conscious ones (Hirschhorn 1990) and that these affect the outcomes of the work undertaken.

Berne (1966) the founder of Transactional Analysis (TA) defined a contract as "an explicit bilateral commitment to a well-defined course of action". He saw contracting as crucial in working relationships and the contract should be transparent, open and mutually agreed by all parties. Berne identified three levels to the contract; the administrative level, the professional level and the psychological level. In TA the psychological contract refers to the underlying dynamics between the parties to the contract. The parties to the contract come to the working relationship with a covert agenda based on beliefs, values, experience, individual frame of reference, fantasy and myths. Thus the challenge in the contracting process is to surface these underlying issues, bring them into the open in such a way as to ensure they do not get in the way of the success of work being undertaken. Berne said that the outcome of the contract will be determined at the psychological level and thus the more aware the individual parties to the contract are of their own beliefs, values, assumptions and covert messages, the less likely these are to get in the way of success.

Micholt (1992) added another dimension to the TA contracting with her introduction of the concept of "psychological distance". She described this as the perceived distance in terms of the relationship that exists between the parties to the contract. In a healthy alliance the relationships are equal, so psychologically the coach, the organisation and the coachee have matching degrees of closeness in relationship terms. Issues arise when any one of the parties feels that the relationships are unbalanced and that closeness exists between two of the parties to the perceived detriment of the third. Micholt suggests that contracts are most likely to get "played out" as conspiratorial or collusive, outside of the awareness of the parties to the contract.

Berne (1961) identified that an organisation has both a public and a private structure. He described the private structure as being formed by the underlying group process and being represented by each person's group imago, which influences 'how one is seen' and 'how one transacts' within the organisation. This links to the concept of the "organisation-in-the-mind".

Coaches must be able to deal with the constant state of tension of being both an insider and an outsider, and of becoming but never achieving the status of family member. Van Poelje suggests that the coach has to be able to achieve what Bowlby (1988) described as a 'secure base' within him/herself and in the organisation within a relatively short space of time. The ability to do this is strongly influenced by the coach's childhood experiences and by the organisational culture.

The challenge is to balance the need for autonomy and affiliation with the need for distance and objectivity. Van Poelje (1994) suggests that most coaches have a background of what she called 'parentified children', good at 'making the family better' and of providing themselves with a secure base in the short term. When this fails in the longer term, however, they fall back on insecure strategies of attachment by either discounting (counter-dependent) or exaggerating (over-dependent) feelings and behaviours. 
The ability of the coach to maintain a balance between attachment and detachment is also challenged by what Van Poelje describes as the 'lure of adjustment'. How can coaches maintain individual boundaries when the need for recognition, structure and intimacy all favour adjustment to the organisational culture? This idea adds another dimension to the comparison between external and internal coaches and how the unconscious may influence the behaviour of the coach.

\section{Looking at the research topic through a TA lens}

The research will be guided by the marriage of the psychoanalytic "organisation-in-the-mind" theory and the TA perspective on contracting, the issues the theory identifies for both internal and external coaches and their capacity to contract as cleanly as is humanly possible. The research will explore the public and private structure of the contracts made by both internal and external coaches and will establish what is or is not accounted for at the psychological level.

The concepts of the "organisation in the mind" and "clean contracting" have to be understood literally and not just metaphorically. They refer not only to the coach's conscious or unconscious mental constructs of the organisation, but also the coaching process, the assumptions he or she makes about aim, task, authority, power, accountability and so forth. It also refers to emotional resonances, registered and present in the mind of the coach.

\section{Aims of the Research}

As coaching has grown as a profession in the last ten years, the volume of writing on the subject has grown exponentially. In the more recent past we have seen the development of core competences for coaches and the accreditation of coach training programmes by professional bodies. Much work has been done on the development of coaching as a profession but it would appear that little attention has been paid to the part that organisational culture plays at an unconscious level in defining the relationship between the coach, the coachee and the client organisation nor the impact this has on the coaching contract and ultimately the achievement of coaching outcomes.

The aim of this project is to ascertain whether coaches are influenced in their professional practice at the unconscious level by the culture of the organisation as they experience it, the extent to which this impacts on the contract and how this differs between internal and external coaches.

This will be a specific piece of research intended to raise awareness in the professional community of the impact of the unconscious on the contracting process in coaching.

The primary research question is "How does the coach's unconscious mind influence the coaching process?"

Objective of the project
The objectives of the project are multi-faceted. The primary objective is to heighten awareness of, and sensitivity to, unconscious processes and how these impact on the outcome of the work undertaken in the coaching relationship.

In pursuit of the primary objective a second objective will be met. This second objective is to ascertain the extent to which organisational, social, relational and psychodynamic influences coalesce to effect individual change.

The third objective is to determine similarities and differences in the 'inner world' of coaches in relation to how they perceive organisational culture.

The final objective is to reflect on the research findings and to consider the ways in which they might inform the on-going development of core competences for coaches and the professional development of this community of practice.

\section{Potential Impact of the Project}

\section{On the researcher's learning}

I fully expected my thoughts and feelings about the subject matter to be challenged and reshaped as the research unfolds and they have been. As I engaged in the data collection and analysis, I asked myself the questions I ask the participants and I expect the answers to shape my practice.

One of the challenges I face is framing the research findings in such a way as to be meaningful to my learning partners whilst at the same time engaging the interest in the wider community of practice.

\section{On the learning of my partners}

Culture in organisations is felt and experienced by all parties to the system and yet it remains intangible. At this stage I believe that whatever the results of the research, there will be key learning for my partners on how the perceived and experienced culture of the organisation is influencing the coaching process. Given that each of the partner organisations regularly engages in a culture survey with its constituent members there may also be information emerging on how coaching is supporting a change in organisational culture.

\section{On the participants}

I anticipated the impact on the participants; both internal and external coaches will be unique to the individual. They were offered the opportunity to critically reflect on their professional practice and each person did this in a different way. I believe that new knowledge will emerged for them through their engagement in the process and that the reflective space generated insights and created awareness both in relation to the coaching process but also in the context of their socially constructed reality. 


\section{On the professional community}

This research project has undoubtedly manifested as a result of my own professional frame of reference, epistemology and ontological perspectives. The primary impact on the community will be a piece of research into a topic previously unexplored within the context of coaching.

How the research will impact on the development of professional practice will depend on the outcomes of the research. The research may confirm that the core competence on "establishing the coaching agreement" and the key behavioural indicators which identify the actions of the coach are sufficient for the purpose of generating "clean" contracts. On the other hand the research may generate data which will encourage the professional community to consider how to increase awareness and measure competence in coaches in the area of self-awareness with particular regard to their inner world and how it is influenced and shaped by the context in which they work.

My experience of the professional community of coaching is that there is an underlying belief in evidence based practice and the research will uncover information to support this.

\section{Ethical Considerations}

\section{Overview}

Exposing hidden social processes puts both the researcher and the research participants at risk, contributing to potentially complex ethical dilemmas. I propose to mitigate the impact of these by first of all creating discourses using ethnographic data from different participants as a means of protecting identities while preserving the authenticity and plausibility of the reported findings. Secondly a framework will be created for selecting which data to present by considering the sense making process. The intention behind this is to enable a balance to be struck between protecting the participants' well-being and my obligations to report findings honestly.

Hammersley \& Atkinson (2007)) argue that ethnographers can be used as a source of data; the reaction of both researcher and participants to change reveals culture. Davies (2007) notes that the researcher's knowledge of his own feelings becomes vital and I believe that my personal and professional journey has created a self-awareness which allows me to account for my own feelings. I am further aware that my process allows for what Schon (1983) termed 'reflection in action' and 'reflection on action'. I recognise that my own background will shape my interpretation and I intend to position myself to acknowledge how my interpretation flows from my cultural, personal and historical experiences. By using a journal to record descriptions and feelings, my reactions will become part of the data used to develop the theory. I will use independent supervision as a process for critical self-reflection and continuing practice development throughout the course of the research.
Participants will be protected during dissemination through not being identified by name or biographical details. I will seek to create coherent discourses from multiple participants and presenting their words through a single character. This will serve the dual purpose of ensuring anonymity when reporting sensitive findings and preserving authenticity. Further this affords protection to the researcher, as a participant, to engage in epistemic reflexivity without endangering others.

\section{Ethical Considerations Arising in this Project}

I consider the core ethical consideration in this project is my potential to become too closely identified with the theoretical framework being used in the research and to therefore unconsciously skew the data thereby reinforcing my own frame of reference.

I am conscious of the time and resource that I am investing in the project and of the non-altruistic reasons for this. Whilst at one level I am interested in conducting the research and offering insights to both my learning partners and the wider community, I also want to be recognised as an 'expert' in the field of psychodynamics of organisational coaching. I have no doubt that the effort required to conduct the research will be worthwhile, I am also mindful that my personal desires could impact in an unhealthy way on how 1 conduct the research and report the findings.

With regard to the participants in the research, I believe there are a number of potential ethical issues, outside of those mentioned in a general sense in 5.1 above. In the first instance there is an issue of mutual consent. By this I mean the extent to which, particularly internal coaches feel free to say whether they want to be involved in the research or not. For me this raises the issue of the psychological contract, for whilst they may be told they have the choice, the question is "do they believe they do?" External coaches may seem to have more freedom of choice but there is the consideration of the extent to which they may over-adapt in an attempt to please the client organisation.

Staying with the potential ethical issues for those who participate in the research, there is the possibility of unspoken fear of being found wanting. The research involves an exploration of the participants' inner world and this may create anxiety in the participants and leave them feeling vulnerable. I am conscious that my own experience of the culture of the organisations who are participating in the research is one where showing vulnerability is not encouraged.

At an organisational level, there may be the potential for the stakeholders to feel threatened by the findings if these show that there is a negative impact on coaching outcomes because of the perceived organisational culture.

A couple of ethical questions arise as I consider the data collection and these are "who owns the data?" and "when and how will it be destroyed after the research is complete?" In my professional role as an OD Consultant it is my view that I own the data and I have agreed with 
the clients a timescale and method for destroying the data after the project is completed. I have less clarity on the answers to these questions in relation to the research project and will seek the advice of my academic supervisor.

And finally, although I am sure that further ethical considerations will emerge as the project unfolds, there is the issue of how the participants and I relate to one another. As a qualified supervisor I will need to pay attention to holding the research process and not moving into supervision of the participant's professional practice.

I believe that my enabling ethical framework, my capacity for self-reflection and my intention to engage in professional supervision will support me to work ethically on this project.

\section{Research Methodologies}

\section{The Researcher's Perspective}

The research will be guided by the marriage of the psychoanalytic "organisation-in-the-mind" theory and the TA perspective on contracting.

I approach my research project from a social constructivist paradigm and although I was introduced to this theory in the context of teaching and learning, it strongly influences my professional practice as an $\mathrm{OD}$ Consultant. We each arrive at our own version of the truth and this is influenced by background, culture and the individual's view of themselves in relation to others and the world. My thinking is influenced by the work of Vygotsky (1978) who suggested that knowledge is first constructed in a social context and then it is appropriated by individuals (Brunning, Schraw \& Ronning 1999). The process of sharing individual perspectives results in individuals constructing understanding that would not be possible alone. This concept informs how I think about the development of organisational culture.

As I consider this in relation to the research project I think that I will be as involved in learning as the participants are. I will compare my version of the truth from my own practice with that of the participants to create a new socially tested version of the truth.

I weave into social constructivism Berne's (1966) theory of the development of personality. So whilst I am drawn to the social constructivist paradigm that social interaction supports the development of language, logic, knowledge and adherence to cultural norms, I also believe that the individual personality is shaped by the child's interpretation of events as he or she experiences these. And, of course, this interpretation is based on intuition and the need for survival because the child does not have adult logic and reasoning capacity. Thus we develop a way of being in the world which serves to help us survive as we perceive it. This way of being becomes our frame of reference and we filter interactions and life experiences in order to fit our frame of reference. We carry this frame of reference into adulthood and thus behave as if on auto-pilot rather than discerning of each situation as we meet it.

Alongside this I think group experience is fundamental. At birth we are introduced into our first, small and intensely personal group, called the family. The group offers the new human being protection and identity. In turn the family is dependent for its survival, identity, beliefs and behaviour on a wide range of formal and informal groups in wider society, school, church, political, friendship, interest, leisure and work groups. So from birth we are enlisted in, and gradually committed to group living in a variety of forms.

Belonging to a group influences us in the following ways:

- Learning

- Attitudes

- Values

- Habits

- Performance and achievement

- Mental and emotional well-being

Our early group experience influences how we engage in wider groups or systems e.g. organisations. We take in to each system with which we engage a preconscious expectation of how that system is or should be. Thus we seek to co-create the social reality that fits our frame of reference.

The body of existing knowledge guiding the research is outlined in section 3 above, primarily Berne's (1996) theory of contracting and Armstrong's (2005) psychoanalysis of organisations. The research will be conducted on the basis of the work of these two writers being propositional knowledge. I am aware I have a deeply held conviction that the work of these two writers is relevant and powerful. So in a sense I am working with concepts which I have accepted as 'my truth'. The ethical considerations in relation to this are covered in section 5 above.

With regard to epistemology, working from a social constructivist perspective, I believe that we co-create knowledge and understanding through social interaction, through a basic human urge to grow and develop, and through a willingness to learn from and be guided by others. We are each of us active players in the learning process and the creation of knowledge. As a learner I tend towards the pragmatist/activist styles (Kolb 1984) with an aversion to taking on theory. Having said that I am drawn to the concept that the body of knowledge we know as theory is co-created in the learning process by a sharing of ideas, testing these out, feeding information back in, and reframing the original ideas in light of the feedback. Within the context of the research project I am working with a body of existing knowledge and the research findings will probably reshape or reframe that knowledge in the light of interpretation of the inner world of the participants.

A sub-question raised by the research project focuses on ontological study; "What does it mean to be human (held within the context of coaching)? Ontological 
learning is learning about being human. The research focuses on generating learning about the human process in the context of the professional role as a coach, human being first, coach second. For me ontological learning investigates key domains of human existence and how they shape our everyday actions and interactions. The ontological learning that will emerge from this research will offer participants, the learning partners and the coaching profession in general a potent means for recreating ourselves individually and collectively.

It is difficult for me to separate epistemology, ontology and personal perspectives. They are closely woven together in my frame of reference and which has implications for my ability to achieve research neutrality. The way I collect the data, what I see, hear and interpret during the data collection, the time of analysis and write-up is influenced by my inner world. Mason (2002) said 'No research or story can be ontologically neutral'.

\section{Approach}

Within the context of qualitative research, the approach has been interpretative phenomenological analysis (IPA). IPA is a recently developed and rapidly growing approach to qualitative inquiry. It originated, and is best known in psychology but is increasingly being picked up by those working in cognate disciplines in the human, social and health sciences. IPA overlaps with other essentially qualitative approaches including ethnography, hermeneutics and symbolic interactionism. Pure phenomenological research seeks essentially to describe rather than explain, and to start from a perspective free from hypotheses or preconceptions (Husserf 1970). More recent humanist and feminist researchers refute the possibility of starting without preconceptions or bias, and emphasise the importance of making clear how interpretations and meanings are placed on findings as well as making the researcher visible in the "frame" of the research as an interested and subjective actor rather than a detached and impartial observer (Stanley \& Wise 1993). This is dealt with in more depth in section 5 of this proposal.

Phenomenology is a philosophical approach to the study of experience. Smith, Flowers and Larkin (2009) state that IPA is concerned with understanding personal lived experience and thus with exploring persons' relatedness to, or involvement in, a particular event or process. According to Smith (1996) IPA has been positioned as an integrative approach. IPA aims to allow the researcher to develop an analytic interpretation of participants' accounts which should be prompted by, and clearly grounded in, but which may also go beyond, the participants own sense making and conceptualizations (Larkin et al 2006).

In choosing this methodology, I committed to exploring, describing, interpreting and situating the means by which the research participants make sense of their experience. A researcher's epistemology according to Holloway (1997), Mason (1996) and Creswell (1994) is literally her theory of knowledge, which serves to decide how the social phenomena will be studied.
In working with IPA methodology and methods I entered the world of the research subjects in an attempt to understand, not simply observe, how they interpret their world and rationalise decisions in the context of the coaching contract. This type of research is a means for exploring and understanding the meaning individuals or groups ascribe to social or human problems. As I have previously mentioned, I will be conducting this research through a particular theoretical lens.

The challenges of conducting an IPA based research project are manifold, not least of which is the generation of large quantities of interview notes, tape recordings and other records which have to be analysed and, of course, the analysis can be challenging when the data does not fall into neat categories. Hycner (1985) and Smith, Flowers and Larkin (2009) give helpful instruction of how to rise to these challenges. Another and significant challenge in conducting this type of research is assessing the validity and quality of the research. This could, of course, be said of all qualitative approaches. The key considerations are outlined by Yardley (2000):

- Sensitivity to context

- Commitment and rigour

- Transparency and coherence

Smith, Flowers and Larkin (2009) offer guidance on how the IPA researcher can take quality and validity seriously and suggest ways in which these can be addressed in an IPA study. I have followed their guiding principles in conducting the research.

I see qualitative research as a form of interpretive enquiry in which I will be making interpretations of what I hear and understand. I have already highlighted that I am aware that my interpretations cannot help but be influenced by my own background, professional development and prior understanding of the subject under review.

Madison (2003) suggests that researchers need to consider what is at stake when they take the role of transmitter of information and skilled interpreter. She suggests there are five central questions for researchers to consider:

- How do we reflect upon and evaluate our own purpose, intentions and frame of analysis as researchers?

- How do we predict the consequences or evaluate our own potential to do harm?

- How do we maintain a dialogue of collaboration in our research projects between ourselves and others?

- How is the specificity of the local story relevant to the broader meanings and operations of the human condition?

- How, in what location or through what intervention, will our work make the greatest contribution to equity, freedom and justice? 
These questions have been key to how I engaged the research methodology.

\section{Considerations and challenges}

I am conscious that the choice of methodology placed me as a key instrument in the process. How I presented myself, how I engaged with participants and how I contracted with them for the conversations impacted on the how safe they felt to engage in the process. My focus in this research has been on eliciting and understanding the meaning that the participants hold about the issue in question. The challenge in contracting for in-depth exploration of the participants' inner worlds has been to show that I have a genuine interest in understanding how they make meaning and how this influences their behaviour. They will needed assurance that I am not blaming and judging them for their way of being or way of working with their clients.

As a Transactional Analyst, I work with Crossman's (1966) concept of permission, protection and potency. In the context of the work I do I see permission as being something that is okay and allowable. For example, it is okay for people to perceive situations as they do and it is okay for them to speak their truth without fear of blame or judgement. In order for a client to take these permissions I have to be perceived as potent, able to hold the process and not to collapse in the face of resistance or strong emotions. I see protection as my ability to co-create a space with my client in which they feel safe and contained. In any research that I have done I recognise the importance of giving the participant permission to be who they are, to speak their truth and to do so without fear of blame or judgement. Alongside this it is crucial not only to offer protection to the individuals involved both in terms of making sure they cannot be identified but also to work on the co-creation of a safe and contained space in which it is safe for us to explore their inner worlds. I have worked with this model in the research interviews.

In the contracting process it was important for me to explain and invite questions from the participants on the research, the role of the researcher and the dissemination of the findings.

\section{Data collection}

As a consequence of taking the IPA approach to this research, certain methods for collecting and analysing data were preferred. Successful data collection strategies require organisation, flexibility and sensitivity. Successful analyses require the systemic application of ideas and methodical rigour; but they also require imagination, playfulness and a combination of reflective critical and conceptual thinking. The researcher engaging in phenomenological inquiry is central to the IPA research focus.

The methods used provided a partial map of the territory which I wished to cross. My aim was to design data collection events which elicited detailed stories, thoughts and feelings from the participants. Semistructured interviews tend to be the preferred method of data collection in IPA research (Reid, Flowers \& Larkin 2005). From my professional experience of conducting research in organisational settings, I believe that one-toone interviews are easily managed, allow a rapport to be developed and give participants the space to think, speak and be heard. My experience is that this type of interview is well suited to in-depth and personal discussion. They also fit with the model of the relationship between researcher and participants that I work with, i.e. that we engaged as equals in a co-created process of inquiry.

I considered and rejected other methods which have been used in IPA such as postal questionnaires, shared electronic mail dialogue and focus groups. The process of interviewing allows the researcher and participants to engage in a dialogue whereby initial questions are modified in the light of participants' responses and the researcher is able to enquire after any other interesting areas which arise.

In considering the data collection process, I further refined my thinking on the boundaries for the study and in particular taking the study beyond semi-structured interviews. I collected information through semistructured interviews in the first instance, then used symbolic representation to invite participants to explore their inner world and moved to indirect observation of the coach in action, inviting the participant to connect what was happening in the coaching process with their symbolic representation

\section{Current Status}

The data collection stage of the project has been completed and I am now in the process of data analysis. I am on schedule to produce the first draft of the thesis by March 2013.

\section{References}

Armstrong, D (2005) - Organisation in the Mind - London Karnac

Berne, E (1961) - Transactional Analysis in PsychotherapyNew York - Grove Press

Berne, E (1966) - Principles of Group Treatment - USA - Oxford University Press

Bowlby, J (1988) - Attachment and Loss - London - Hogarth

Bruning, R H, Schraw, G J, \& Ronning, R R, (1999) Cognitive Psychology and Instruction $3^{\text {rd }}$ edition - New Jersey - Prentice Hall.

Creswell, J W (1994) - Research Design: Qualitative and Quantitative Approaches - London - Sage

Crossman, P (1966) - Permission and Protection Transactional Analysis Bulletin 5: (19) - 152-4

Davies, C A (2007) - Reflexive Ethnography: A Guide to Researching Self and Others - London - Routledge

Hammersley, M \& Atkinson, P (2007) - Ethnography: Principles in Practice - London - Tavistock

Hirschhorn, L (1990) - The Workplace Within: The Psychodynamics of Organisational Life - USA - MIT Press 
Holloway, I. (1997). Basic concepts for qualitative research. Oxford: Blackwell Science.

Husserf, E (1970) - Logical Investigations - New York Humanities Press (translated by D Carr)

Hutton, J, Balgazette, J, \& Reed, B (1997) - Organisation-inthe-mind - In - J E Neumann, K Kellner \& A Dawson-Shepherd (Eds.) - Developing Organisational Consultancy (pp 113-116) London - Routledge

Hycner, R H (1985) "Some guidelines for the phenomenological analysis of interview data," Human Studies 8, 279-303

Larkin, M, Watts, S \& Clifton, E (2006) - Giving voice and making sense in Interpretative Phenomenological Analysis Qualitative Research in Psychology - 3: 102 -120

Madison, D S (2003) - Critical Ethnography: Method, Ethics and Performance - London - Sage

Mason, J. (1996). Qualitative researching. London: Sage.

Mason, J (2002) - Qualitative Researching $2^{\text {nd }}$ edition - London - Sage

Micholt, N (1992) Psychological Distance and Group Intervention - Transactional Analysis Journal: (6) 22-4

Peltier, B 2009 - The Psychology of Executive Coaching $2^{\text {nd }}$ Edition - New York - Routledge
Shapiro, E R \& Carr, A W (1991) - Lost in Familiar Places:

Creating New Connections Between the Individual and Society - New Jersey - Yale University Press

Smith, J A (2009) - Hermeneutics, human sciences and health: Linking theory and practice - International Journal of Qualitative Studies on Health and Well-Being - 2: 3-11

Smith, J A; Flowers, P \& Larkin, M (2009) - Interpretative Phenomenological Analysis: Theory, Method and Research London - Sage

Stanley, L \& Wise, S (1993) - Breaking Out Again: Feminist Ontology and Epistemology - London - Routledge

St John Brooks, K (2009) - Moral Support - Coaching At Work: Vol 5: Issue 1 - Chartered Institute of Personnel and Development

Tyler, K (2000) - Scoring Big in the Workplace - HR Magazine 45(6): 96-106

Van Poelje, S (1994) - Contracting for Organisational Change European Association of Transactional Analysis Conference Maastricht Papers

Wilson, S (2008) - True Dilemmas - Coaching At Work: Vol 3: Issue 3 - Chartered Institute of Personnel and Development

Yardley, L (2000) - Dilemmas in qualitative health research Psychology and Health - 15: 215-228 